\title{
General linearization formulae for products of continuous hypergeometric-type polynomials
}

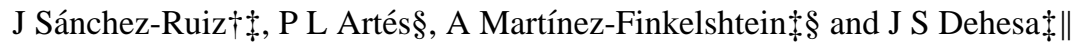 \\ $\dagger$ Departamento de Matemáticas, Universidad Carlos III de Madrid, Avda. de la Universidad 30 \\ 28911 Leganés, Madrid, Spain \\ \$ Instituto Carlos I de Física Teórica y Computacional, Universidad de Granada, 18071 Granada, \\ Spain \\ § Departamento de Estadística y Matemática Aplicada, Universidad de Almería, La Cañada, \\ 04120 Almería, Spain \\ || Departamento de Física Moderna, Universidad de Granada, 18071 Granada, Spain
}

\begin{abstract}
The linearization of products of wavefunctions of exactly solvable potentials often reduces to the generalized linearization problem for hypergeometric polynomials (HPs) of a continuous variable, which consists of the expansion of the product of two arbitrary HPs in series of an orthogonal HP set. Here, this problem is algebraically solved directly in terms of the coefficients of the second-order differential equations satisfied by the involved polynomials. General expressions for the expansion coefficients are given in integral form, and they are applied to derive the connection formulae relating the three classical families of hypergeometric polynomials orthogonal on the real axis (Hermite, Laguerre and Jacobi), as well as several generalized linearization formulae involving these families. The connection and linearization coefficients are generally expressed as finite sums of terminating hypergeometric functions, which often reduce to a single function of the same type; when possible, these functions are evaluated in closed form. In some cases, sign properties of the coefficients such as positivity or non-negativity conditions are derived as a by-product from their resulting explicit representations.
\end{abstract}

\section{Introduction}

Consider the second-order differential operator

$$
\mathcal{F}[y](x)=\sigma(x) y^{\prime \prime}(x)+\tau(x) y^{\prime}(x)
$$

where $\sigma(x)$ and $\tau(x)$ are polynomials whose degrees are not greater than two and one, respectively. If $\left|\tau^{\prime}\right|+\left|\sigma^{\prime \prime}\right| \neq 0$, then for every $n \in \mathbb{N}, \mathcal{F}$ has a polynomial eigenfunction $y(x)=y_{n}(x)$ of degree $n$ corresponding to the eigenvalue

$$
\lambda_{n}=n \tau^{\prime}+{ }_{2}^{1} n(n-1) \sigma^{\prime \prime} .
$$

These polynomials are usually called hypergeometric-type polynomials (or continuous hypergeometric-type polynomials, in contrast with those which appear as eigenfunctions of second-order linear difference operators); by means of linear changes of the variable, they can be reduced to one of the four classical families (Hermite, Laguerre, Jacobi and Bessel). The hypergeometric-type polynomials are involved in the classical eigenfunctions of singular Sturm-Liouville equations [1-6] as well as in the quantum mechanical wavefunctions of the exactly solvable potentials [5,7-10], and they are used in the mathematical modelling of a great amount of physical and chemical phenomena [3, 5, 11-14]. 
This paper deals with the general hypergeometric linearization problem, which is the problem of finding the coefficients $g_{n m k}$ in the expansion of the product of two (hypergeometric) polynomials, $p_{n}(x) q_{m}(x)$, in terms of an arbitrary sequence of orthogonal hypergeometric polynomials $\left\{y_{k}(x)\right\}$,

$$
p_{n}(x) q_{m}(x)=\sum_{k=0}^{n+m} g_{n m k} y_{k}(x) .
$$

Note that, in this setting, the polynomials $p_{n}, q_{m}$ and $y_{k}$ may belong to three different hypergeometric families. Particular cases of this problem have been matter of intensive research (see, e.g., $[12,15]$ and the bibliography therein), and frequently receive different names. For example, when the polynomials $p_{n}, q_{m}$ and $y_{k}$ are solutions of the same differential equation (1), this is usually called the (standard) linearization [12] or Clebsch-Gordan-type problem [16] for hypergeometric polynomials (the name Clebsch-Gordan is attached because the structure is similar to the Clebsch-Gordan series for spherical functions [17]). On the other hand, taking $q_{m}(x)=1$ in (2), we are faced with the so-called connection problem, which for $p_{n}(x)=x^{n}$ is known as the inversion problem for the family $\left\{y_{k}(x)\right\}$.

The literature on the standard linearization and connection problems is extremely vast, and a variety of methods and approaches for computing the coefficients $g_{n m k}$ in (2) have been developed. For the classical families of polynomials, explicit expressions have been obtained, usually in terms of generalized hypergeometric series, exploiting with this purpose several of their characterizing properties: recurrence relations, generating functions, orthogonality weights, etc (see, e.g., [3,12,16,18-20]). Another, rather general, approach allows computation of the standard linearization and connection coefficients recursively (see, e.g., [15, 20, 21]). In contrast, the general linearization problem has not yet been solved $[12,15]$, although some partial results are known for Jacobi polynomials [14], and explicit expressions for the coefficients $g_{n m k}$ when $p_{n}, q_{m}$ and $y_{k}$ are Laguerre polynomials with different parameters have also been given [16]. This is somewhat surprising, because there are numerous fundamental and applied questions related to this problem, such as the linearization of products of basis-set functions associated to shape-invariant potentials [10], the transformation formulae between quantum mechanical wavefunctions in different coordinate systems (e.g. the bound-state wavefunctions on $\mathrm{L}^{2}\left(\mathbb{R}^{3}\right)$ in spherical and parabolic coordinates [22]), the interbasis expansions for potentials of equal [23] and different [24] dimensionality (e.g. the passage formulae from the $\mathbb{R}^{3}$ hydrogen wavefunctions to $\mathbb{R}^{4}$ oscillator wavefunctions [22]), the determination of the Talmi-Brody-Moshinsky coefficients [25] so widely used in nuclear structure, and the evaluation of two-centre, two- and three-electron integrals in variational atomic analysis [13].

The main aim of this paper is to produce some analytic (in general, integral) representations for the coefficients $g_{n m k}$ in (2), extending the method proposed very recently by the authors [26] to solve the inversion, connection, and standard linearization problems for hypergeometric polynomials. These analytic expressions of $g_{n m k}$ are given directly in terms of the coefficients of the differential equations corresponding to the hypergeometric polynomials $p_{n}, q_{n}$ and $y_{n}$, which is often a desirable feature. In fact, products of hypergeometric polynomials appear naturally when dealing with eigenfunction systems of Sturm-Liouville equations $[27,28]$ and the Schrödinger equations associated to central potentials [7,17]. Furthermore, the quantum mechanical equation of motion of numerous physical systems with a shape-invariant potential can be reduced to a second-order differential equation of hypergeometric character $[5,7,8]$.

In many applications of orthogonal polynomials, it is often important to know whether the linearization and connection coefficients are positive or non-negative (see, e.g., [12, 29,30]). During the last decades, several sufficient conditions for these sign properties to hold have been derived, both for general polynomials and for the classical families (see, e.g., [12,30-34]). 
Although the focus of the present paper is on the explicit computation of the coefficients, rather than on the study of their sign properties, we shall find that in many cases these sign properties become obvious from the representations given here; typically, this happens when the coefficients can be written as a sum of terms which are shown to have the same sign by taking into account the comments after equation (A.1).

The paper is structured as follows. First, in section 2, the general expressions for the linearization coefficients are obtained, and the particular cases of the standard linearization and connection problems are singled out. We also find the remarkable result that, for hypergeometric polynomials, the linearization coefficients can always be written as a finite combination of connection coefficients. Later, for illustration, the connection formulae relating the three classical families of hypergeometric polynomials orthogonal on the real line (Hermite, Laguerre and Jacobi) are obtained (section 3). The linearization problems for Hermite and Laguerre polynomials are revisited, and closed expressions are also obtained for the coefficients of the Hermite expansion of products involving Hermite polynomials (section 4). Some concluding remarks are given in section 5 , and, finally, the definitions and identities for special functions used throughout the paper are collected in the appendix.

\section{General results}

We begin by introducing some notation. Below $\mathbb{P}$ is the class of all polynomials with real coefficients. If $\left\{y_{n}(x)\right\}_{n \in \mathbb{N}}$ stands for the sequence of monic hypergeometric polynomials corresponding to operator (1), then we denote by $y_{n, k}(x)$, with $n, k \in \mathbb{N}$, the monic polynomial eigenfunction of degree $n$ of the operator

$$
\begin{gathered}
\mathcal{F}_{k}[y](x)=\sigma(x) y^{\prime \prime}(x)+\tau_{k}(x) y^{\prime}(x) \quad \tau_{k}(x)=\tau(x)+k \sigma^{\prime}(x) \\
\text { with }\left|\tau_{k}^{\prime}\right|+\left|\sigma^{\prime \prime}\right| \neq 0 \text {, so that } \\
y_{n}(x)=y_{n, 0}(x) \quad y_{n, k}^{\prime}(x)=n y_{n-1, k+1}(x) .
\end{gathered}
$$

Analogous notation will be used for other sequences of monic hypergeometric polynomials as well. For the sake of brevity, below we omit the subindex $k$ when $k=0$.

An explicit expression for these polynomials is provided by the Rodrigues formula. Fix

$\omega(x)=\exp \left(\int^{x} \frac{\tau(t)-\sigma^{\prime}(t)}{\sigma(t)} \mathrm{d} t\right) \quad \omega_{k}(x)=[\sigma(x)]^{k} \omega(x) \quad k \geqslant 0$.

Then $\omega_{k}(x)$ is a solution of the so-called Pearson's equation, $\left[\sigma(x) \omega_{k}(x)\right]^{\prime}=\tau_{k}(x) \omega_{k}(x)$. If

$$
A_{n, k}=\prod_{j=0}^{n-1}\left(\tau_{k}^{\prime}+\frac{n+j-1}{2} \sigma^{\prime \prime}\right)^{-1}=\prod_{j=0}^{n-1}\left(\tau^{\prime}+\frac{n+j+2 k-1}{2} \sigma^{\prime \prime}\right)^{-1}
$$

then

$$
y_{n, k}(x)=\frac{A_{n, k}}{\omega_{k}(x)} \frac{\mathrm{d}^{n} \omega_{n+k}(x)}{\mathrm{d} x^{n}} .
$$

In what follows, we assume, additionally, the existence of two values $-\infty \leqslant a<b \leqslant \infty$ such that

$$
\omega(x) \in C(a, b) \quad \lim _{x \rightarrow a+} \omega_{1}(x) x^{k}=\lim _{x \rightarrow b-} \omega_{1}(x) x^{k}=0 \quad k \geqslant 0 .
$$

This assumption leads to orthogonality of $\left\{y_{n}(x)\right\}$ with respect to the weight function $\omega(x)$ on the interval $[a, b]$ (see [5]),

$$
\int_{a}^{b} y_{n}(x) y_{m}(x) \omega(x) \mathrm{d} x=\varkappa_{n} \delta_{n, m} \quad \varkappa_{n}=(-1)^{n} n ! A_{n} \gamma_{n}
$$


where $\left\{\gamma_{n}\right\}$ denotes the sequence of generalized moments of $\omega(x)$,

$$
\gamma_{n}=\int_{a}^{b} \omega_{n}(x) \mathrm{d} x .
$$

Likewise, every sequence $\left\{y_{n, k}(x)\right\}$ is then orthogonal with respect to the weight function $\omega_{k}(x)$ on the same interval,

$$
\int_{a}^{b} y_{n, k}(x) y_{m, k}(x) \omega_{k}(x) \mathrm{d} x=\varkappa_{n, k} \delta_{n, m} \quad \varkappa_{n, k}=(-1)^{n} n ! A_{n, k} \gamma_{n+k} .
$$

Let us denote by $\left\{p_{n}(x)\right\}$ and $\left\{q_{m}(x)\right\}$ two (possibly different) polynomial sequences, not necessarily orthogonal or hypergeometric. The orthogonality relation (9) for the sequence $\left\{y_{n}(x)\right\}$ leads to the following explicit expression for the Fourier coefficients $g_{n m k}$ of the general linearization formula (2):

$$
g_{n m k}=\frac{1}{\varkappa_{k}} \int_{a}^{b} p_{n}(x) q_{m}(x) y_{k}(x) \omega(x) \mathrm{d} x .
$$

This equation enables us to compute $g_{n m k}$ in a straightforward way provided that the explicit expressions of the three polynomials involved are known. However, as we shall show in the following, the hypergeometric nature of the polynomials can be used to derive alternative expressions for the linearization coefficients, which may be written in terms of the coefficients of the underlying differential operators and are computationally more efficient.

Taking advantage of equations (7) and (9), we can rewrite (12) as

$$
g_{n m k}=\frac{(-1)^{k}}{k ! \gamma_{k}} \int_{a}^{b} p_{n}(x) q_{m}(x) \frac{\mathrm{d}^{k} \omega_{k}(x)}{\mathrm{d} x^{k}} \mathrm{~d} x .
$$

It is easy to verify that for every $P \in \mathbb{P}$ there exists a $Q \in \mathbb{P}$ such that

$$
\frac{\mathrm{d}^{j}}{\mathrm{~d} x^{j}}\left[\omega_{k}(x) P(x)\right]=\omega_{k-j}(x) Q(x) \quad j \leqslant k .
$$

Thus, integrating by parts $k$ times and taking into account the boundary conditions (8), Leibniz's rule allows us to rewrite (13) in the form

$$
g_{n m k}=\frac{1}{k ! \gamma_{k}} \sum_{j=j_{-}}^{j_{+}}\left(\begin{array}{l}
k \\
j
\end{array}\right) \int_{a}^{b} \frac{\mathrm{d}^{j} p_{n}(x)}{\mathrm{d} x^{j}} \frac{\mathrm{d}^{k-j} q_{m}(x)}{\mathrm{d} x^{k-j}} \omega_{k}(x) \mathrm{d} x
$$

where $j_{-}=\max (0, k-m), j_{+}=\min (k, n)$.

In comparison with (12), (14) has two main advantages. Firstly, it does not require the knowledge of the explicit expression of the polynomials $y_{k}(x)$. Secondly, although in both cases $g_{n m k}$ is expressed as a three-level summation of terms which are essentially the moments of a weight function, the degree reduction by derivatives provides a smaller number of terms when (14) is used. For instance, the number of terms in the expressions for $g_{n n n}$ given by (12) and (14) are, respectively, $(n+1)^{3}$ and

$$
\sum_{j=0}^{n}(n-j+1)(j+1)=\frac{1}{6}(n+1)(n+2)(n+3) .
$$

Equation (14) is especially useful when the derivatives of the polynomials $p_{n}(x)$ and $q_{m}(x)$ have simple expressions. In particular, taking $p_{n}(x)=x^{n}$ and $q_{m}(x)=1$, we can obtain a solution for the inversion problem in terms of the moments of the weights $\omega_{k}(x)$ [26]: the coefficient $\iota_{n k}$ in the expansion

$$
x^{n}=\sum_{k=0}^{n} \iota_{n k} y_{k}(x)
$$


is given by

$$
\iota_{n k}=\left(\begin{array}{l}
n \\
k
\end{array}\right) \frac{1}{\gamma_{k}} \int_{a}^{b} x^{n-k} \omega_{k}(x) \mathrm{d} x .
$$

Let us assume now that both $p_{n}(x)$ and $q_{m}(x)$ are also monic polynomials of hypergeometric type. Formula (14) can then be written, using (4), in the form

$$
g_{n m k}=\frac{1}{\gamma_{k}} \sum_{j=j_{-}}^{j_{+}}\left(\begin{array}{c}
n \\
j
\end{array}\right)\left(\begin{array}{c}
m \\
k-j
\end{array}\right) \mathcal{I}_{n m k}(j) \quad \mathcal{I}_{n m k}(j)=\int_{a}^{b} p_{n-j, j}(x) q_{m+j-k, k-j}(x) \omega_{k}(x) \mathrm{d} x .
$$

This equation is feasible for the computation of the generalized linearization coefficients whenever the explicit expressions of the polynomials involved in the integral are known, as is the case, e.g., for the classical hypergeometric families (see the appendix). For general families of polynomials, when only the coefficients of the corresponding differential operators are available, we can make one more step and find an equivalent expression for $g_{n m k}$ that does not require the knowledge of the explicit expressions of the polynomials. Initially, we restrict our attention to the standard linearization and connection problems, for which simpler formulae can be derived from (15).

In the particular case when the three families of hypergeometric polynomials coincide, we have a solution for the (standard) linearization (or Clebsch-Gordan) problem. Using the orthogonality property (9), equation (2) simplifies to

$$
y_{n}(x) y_{m}(x)=\sum_{k=|n-m|}^{n+m} l_{n m k} y_{k}(x)
$$

and (15) now reads as

$l_{n m k}=\frac{1}{\gamma_{k}} \sum_{j=j_{-}}^{j_{+}}\left(\begin{array}{c}n \\ j\end{array}\right)\left(\begin{array}{c}m \\ k-j\end{array}\right) \mathcal{I}_{n m k}(j) \quad \mathcal{I}_{n m k}(j)=\int_{a}^{b} y_{n-j, j}(x) y_{m+j-k, k-j}(x) \omega_{k}(x) \mathrm{d} x$.

Using equation (7) for $y_{n-j, j}(x)$, the previous expression for the integrals $\mathcal{I}_{n m k}(j)$ can be written as

$$
\mathcal{I}_{n m k}(j)=A_{n-j, j} \int_{a}^{b} \frac{\mathrm{d}^{n-j} \omega_{n}(x)}{\mathrm{d} x^{n-j}} y_{m+j-k, k-j}(x)[\sigma(x)]^{k-j} \mathrm{~d} x .
$$

Observe that by (4) and (7), for $0 \leqslant j \leqslant n$,

$$
\frac{\mathrm{d}^{j}}{\mathrm{~d} x^{j}}\left(\frac{1}{\omega_{k}(x)} \frac{\mathrm{d}^{n} \omega_{n+k}(x)}{\mathrm{d} x^{n}}\right)=\frac{n !}{(n-j) !} \frac{A_{n-j, k+j}}{A_{n, k}} \frac{1}{\omega_{k+j}(x)} \frac{\mathrm{d}^{n-j} \omega_{n+k}(x)}{\mathrm{d} x^{n-j}} .
$$

Thus, integrating by parts $n-j$ times and taking into account the boundary conditions (8),

$$
\mathcal{I}_{n m k}(j)=(-1)^{n-j} A_{n-j, j} \int_{a}^{b} \omega_{n}(x) \frac{\mathrm{d}^{n-j}}{\mathrm{~d} x^{n-j}}\left(y_{m+j-k, k-j}(x)[\sigma(x)]^{k-j}\right) \mathrm{d} x .
$$

Using the Leibniz rule and equation (4),

$$
\begin{aligned}
\mathcal{I}_{n m k}(j)=(-1)^{n-j} A_{n-j, j} \sum_{i=i_{-}}^{i_{+}}\left(\begin{array}{c}
n-j \\
i
\end{array}\right) \frac{(m-k+j) !}{(m-n-k+2 j+i) !} \\
\times \int_{a}^{b} y_{m-n+2 j-k+i, k-2 j+n-i}(x) \frac{\mathrm{d}^{i}[\sigma(x)]^{k-j}}{\mathrm{~d} x^{i}} \omega_{n}(x) \mathrm{d} x
\end{aligned}
$$


where $i_{-}=\max (0, n-m+k-2 j), i_{+}=\min \{n-j,(k-j) \operatorname{deg}[\sigma(x)]\}$. Now we can substitute the explicit expression of the remaining polynomial, given by the Rodrigues formula (7), and integrate by parts again, which yields

$$
\begin{gathered}
\mathcal{I}_{n m k}(j)=(-1)^{m+k+j} A_{n-j, j} \sum_{i=i_{-}}^{i_{+}}\left(\begin{array}{c}
n-j \\
i
\end{array}\right) \frac{(-1)^{i}(m-k+j) !}{(m-n-k+2 j+i) !} A_{m-n+2 j+i-k, k-2 j+n-i} \\
\times \int_{a}^{b} \omega_{m}(x) \frac{\mathrm{d}^{m-n+2 j-k+i}}{\mathrm{~d} x^{m-n+2 j-k+i}}\left([\sigma(x)]^{2 j-k+i} \frac{\mathrm{d}^{i}[\sigma(x)]^{k-j}}{\mathrm{~d} x^{i}}\right) \mathrm{d} x .
\end{gathered}
$$

In spite of its apparent complexity, this formula has the advantage that no derivatives of the weight functions are involved; it does not make use of the explicit expressions of the polynomials either. In fact, if we know $\sigma(x)$ we can easily express the integrals appearing in (18) as a linear combination of the moments of the weight function $\omega_{m}(x)$, which makes this equation suitable for symbolic manipulation.

Let us consider now the connection problem

$$
p_{n}(x)=\sum_{k=0}^{n} c_{n k} y_{k}(x)
$$

where $\left\{p_{n}(x)\right\}$ is the sequence of monic polynomial eigenfunctions of the operator

$$
\mathcal{G}[y](x)=\tilde{\sigma}(x) y^{\prime \prime}(x)+\tilde{\tau}(x) y^{\prime}(x)
$$

and $\operatorname{deg}\left[p_{n}(x)\right]=n$. Taking into account that $q_{0}(x)=1$ for any sequence $\left\{q_{n}(x)\right\}$ of monic polynomials, we readily see that $c_{n k}=g_{n 0 k}$, so that the connection coefficients $c_{n k}$ can be obtained as the particular case $m=0$ of both (12) and (15). Again, it turns out to be much more convenient to use (15), which leads to

$$
c_{n k}=\frac{1}{\gamma_{k}}\left(\begin{array}{l}
n \\
k
\end{array}\right) \mathcal{I}_{n k} \quad \mathcal{I}_{n k}=\int_{a}^{b} p_{n-k, k}(x) \omega_{k}(x) \mathrm{d} x .
$$

Using this formula, $c_{n k}$ is expressed as a simple summation with $n-k+1$ terms, while (12) would give a double summation with $(n+1)(k+1)$ terms. Furthermore, $(21)$ does not require the use of the explicit expression of $y_{k}(x)$.

By equation (7), the previous formula for $\mathcal{I}_{n k}$ can be written as

$$
\mathcal{I}_{n k}=\tilde{A}_{n-k, k} \int_{a}^{b} \frac{\omega_{k}(x)}{\tilde{\omega}_{k}(x)} \frac{\mathrm{d}^{n-k} \tilde{\omega}_{n}(x)}{\mathrm{d} x^{n-k}} \mathrm{~d} x
$$

or, equivalently, integrating by parts $n-k$ times,

$$
\mathcal{I}_{n k}=(-1)^{n-k} \tilde{A}_{n-k, k} \int_{a}^{b} \tilde{\omega}_{n}(x) \frac{\mathrm{d}^{n-k}}{\mathrm{~d} x^{n-k}}\left(\frac{\omega_{k}(x)}{\tilde{\omega}_{k}(x)}\right) \mathrm{d} x .
$$

A common situation in connecting polynomials of the same family, but with different parameters, is when $\tilde{\sigma}(x)=\sigma(x)$. In this case, if we put $\tilde{\omega}(x)=f(x) \omega(x)$, the previous equation takes the form

$$
\mathcal{I}_{n k}=(-1)^{n-k} \tilde{A}_{n-k, k} \int_{a}^{b} f(x) \omega_{n}(x) \frac{\mathrm{d}^{n-k}}{\mathrm{~d} x^{n-k}}\left(\frac{1}{f(x)}\right) \mathrm{d} x
$$

which may be useful if the derivatives of $1 / f(x)$ have simple expressions.

For arbitrary families of polynomials, the generalized linearization problem can always be reduced to a combination of two connection and one standard linearization problems: if we 
write

$$
\begin{aligned}
& p_{n}(x)=\sum_{s=0}^{n} c_{n s}(p) y_{s}(x) \quad q_{m}(x)=\sum_{t=0}^{m} c_{m t}(q) y_{t}(x) \\
& y_{s}(x) y_{t}(x)=\sum_{k=|s-t|}^{s+t} l_{s t k} y_{k}(x)
\end{aligned}
$$

then we have

$$
p_{n}(x) q_{m}(x)=\sum_{s=0}^{n} \sum_{t=0}^{m} \sum_{k=|s-t|}^{s+t}\left(c_{n s}(p) c_{m t}(q) l_{s t k}\right) y_{k}(x)
$$

so that the generalized linearization coefficients $g_{n m k}$ in (2) may be computed as

$$
g_{n m k}=\sum_{|s-t| \leqslant k \leqslant s+t} c_{n s}(p) c_{m t}(q) l_{s t k} .
$$

It is a remarkable fact that, in the case when all the involved polynomials are of hypergeometric type, equation (15) enables us to express the linearization coefficients in terms only of two connection coefficients, namely those corresponding to the expansions of the polynomials $p_{n-j, j}(x)$ and $q_{m+j-k, k-j}(x)$ in series of the $\left\{y_{r, k}(x)\right\}$,

$p_{n-j, j}(x)=\sum_{r=0}^{n-j} c_{n-j, r}^{(j, k)}(p) y_{r, k}(x) \quad q_{m+j-k, k-j}(x)=\sum_{s=0}^{m+j-k} c_{m+j-k, s}^{(k-j, k)}(q) y_{s, k}(x)$.

Substituting these expressions into (15) and using the orthogonality relation (11), we obtain

$$
\begin{aligned}
& g_{n m k}=\frac{1}{\gamma_{k}} \sum_{j=j_{-}}^{j_{+}}\left(\begin{array}{c}
n \\
j
\end{array}\right)\left(\begin{array}{c}
m \\
k-j
\end{array}\right) \mathcal{I}_{n m k}(j) \\
& \mathcal{I}_{n m k}(j)=\sum_{r=0}^{r_{+}} c_{n-j, r}^{(j, k)}(p) c_{m+j-k, r}^{(k-j, k)}(q) \varkappa_{r, k}
\end{aligned}
$$

where $r_{+}=\min (n-j, m+j-k)$. In particular, for the standard linearization coefficients $l_{n m k}$ given by (17) the previous formula does apply with $p=q=y$, and we can omit the arguments of the connection coefficients to simplify the notation.

Next, we shall illustrate the application of the general results given in this section by using them to find explicit expressions for the coefficients in the connection formulae relating the three classical families of monic polynomials, orthogonal on the real axis (Hermite, Laguerre and Jacobi), as well as in several linearization formulae involving these families. In fact, as a rule we give two different expressions for each set of coefficients: namely, as a sum of binomial type and as a hypergeometric series (see equation (A.2)); the conversion of the former into the latter has been either carried out or checked using the Mathematica package HYP by C Krattenthaler [35]. All the necessary definitions and well known identities are gathered in appendix A.1, which we shall often make use of without explicit reference to it.

\section{Connection formulae}

Leaving aside the trivial case of the Hermite-Hermite connection, for which we simply have $c_{n k}=\delta_{n, k}$, there are eight connection formulae of the form (19) relating the Hermite, Laguerre and Jacobi families of orthogonal polynomials. In this section, we obtain explicit expressions for the connection coefficients of these eight problems using equations (21)-(24). Most of these expressions are already known in the literature, while others seem to be new; in the former case, references where the results can be found are indicated. Likewise, whenever we find sign properties for the coefficients that are already known, the corresponding references are also indicated. 


\subsection{Expansions in series of Hermite polynomials}

Following from (21) and (22),

$$
p_{n}(x)=\sum_{k=0}^{n} c_{n k} H_{k}(x) \quad c_{n k}=\frac{1}{\sqrt{\pi}}\left(\begin{array}{l}
n \\
k
\end{array}\right) \mathcal{I}_{n k}
$$

where

$$
\mathcal{I}_{n k}=\int_{-\infty}^{\infty} p_{n-k, k}(x) \mathrm{e}^{-x^{2}} \mathrm{~d} x=\tilde{A}_{n-k, k} \int_{-\infty}^{\infty} \frac{\mathrm{d}^{n-k} \tilde{\omega}_{n}(x)}{\mathrm{d} x^{n-k}} \frac{\mathrm{e}^{-x^{2}}}{\tilde{\omega}_{k}(x)} \mathrm{d} x
$$

3.1.1. Connection with Laguerre polynomials. For $p_{n}(x)=L_{n}^{(\alpha)}(x)$, the second expression for $\mathcal{I}_{n k}$ in (30) reads

$$
\mathcal{I}_{n k}=(-1)^{n-k} \int_{-\infty}^{\infty} \frac{\mathrm{d}^{n-k}\left(x^{\alpha+n} \mathrm{e}^{-x}\right)}{\mathrm{d} x^{n-k}} \frac{\mathrm{e}^{-x^{2}}}{x^{\alpha+k} \mathrm{e}^{-x}} \mathrm{~d} x .
$$

Using Leibniz's rule to evaluate the derivative in the right-hand side, together with the well known moments of the weight $\exp \left(-x^{2}\right)$, we find that

$$
\mathcal{I}_{n k}=(-1)^{n-k} \Gamma(n+\alpha+1) \sum_{j=0}^{[(n-k) / 2]}\left(\begin{array}{c}
n-k \\
2 j
\end{array}\right) \frac{\Gamma\left(j+\frac{1}{2}\right)}{\Gamma(k+2 j+\alpha+1)}
$$

where, as usual, the square brackets denote integer part of the expression within. The same result can be obtained more easily by substituting in the first formula of (30) the explicit expression of $p_{n-k, k}(x)=L_{n-k}^{(\alpha+k)}(x)$ given in (A.11). Thus, with account of (29), we obtain $(\mathrm{cf}[18, \mathrm{p} 216])$

$$
\begin{aligned}
c_{n k} & =(-1)^{n-k}\left(\begin{array}{l}
n \\
k
\end{array}\right) \sum_{j=0}^{[(n-k) / 2]}\left(\begin{array}{c}
n-k \\
2 j
\end{array}\right)\left(\frac{1}{2}\right)_{j}(k+2 j+\alpha+1)_{n-k-2 j} \\
& =(-1)^{n-k}(k+\alpha+1)_{n-k}\left(\begin{array}{l}
n \\
k
\end{array}\right){ }_{2} F_{2}\left(\begin{array}{c}
\frac{k-n}{2}, \frac{k-n+1}{2} \\
\frac{k+\alpha+1}{2}, \frac{1}{2}+1
\end{array} \mid \frac{1}{4}\right) .
\end{aligned}
$$

From the first of these expressions, we readily see that the sign of $c_{n k}$ is $(-1)^{n-k}$.

3.1.2. Connection with Jacobi polynomials. For $p_{n}(x)=P_{n}^{(\alpha, \beta)}(x)$, application of Leibniz's rule to the second expression for $\mathcal{I}_{n k}$ in (30) gives

$$
\begin{gathered}
\mathcal{I}_{n k}=\frac{(-1)^{n-k}}{(n+k+\alpha+\beta+1)_{n-k}} \sum_{j=0}^{n-k}(n-j+\alpha+1)_{j}(k+j+\beta+1)_{n-k-j} \\
\times(-1)^{j} \int_{-\infty}^{\infty} B_{n-k, j}(x) \mathrm{e}^{-x^{2}} \mathrm{~d} x
\end{gathered}
$$

where $B_{n-k, j}(x)=\left(\begin{array}{c}n-k \\ j\end{array}\right)(1-x)^{n-k-j}(1+x)^{j}$ is a Bernstein polynomial. Taking advantage of the properties of $B_{n, k}(x)$, it is easy to expand the last integral as a linear combination of the moments of the weight $\exp \left(-x^{2}\right)$ or to compute it recursively. However, a simpler result can be obtained using in the first formula of (30) the explicit expression of $p_{n-k, k}(x)=P_{n-k}^{(\alpha+k, \beta+k)}(x)$ given by (A.12), which leads to

$$
\mathcal{I}_{n k}=\sum_{j=0}^{n-k}\left(\begin{array}{c}
n-k \\
j
\end{array}\right) \frac{2^{n-k-j}(k+j+\alpha+1)_{n-k-j}}{(n+k+j+\alpha+\beta+1)_{n-k-j}} \int_{-\infty}^{\infty}(x-1)^{j} \mathrm{e}^{-x^{2}} \mathrm{~d} x .
$$


Evaluating the integrals in the right-hand side, the expression for $c_{n k}$ follows in a straightforward way from (29):

$c_{n k}=\left(\begin{array}{l}n \\ k\end{array}\right) \sum_{j=0}^{n-k}\left(\begin{array}{c}n-k \\ j\end{array}\right) \frac{(-1)^{j} 2^{n-k-j}(k+j+\alpha+1)_{n-k-j}}{(n+k+j+\alpha+\beta+1)_{n-k-j}} \sum_{m=0}^{[j / 2]}\left(\begin{array}{c}j \\ 2 m\end{array}\right)\left(\frac{1}{2}\right)_{m}$.

The sum over $m$ can be written as a ${ }_{2} F_{0}$ hypergeometric function of unit argument and upper parameters $-j / 2,(1-j) / 2$. Alternatively, interchanging the order of summation and shifting the index $j$ to $l=j-2 m$, the previous formula reads

$$
\begin{aligned}
c_{n k}=\left(\begin{array}{l}
n \\
k
\end{array}\right) & \sum_{m=0}^{[(n-k) / 2]}\left(\begin{array}{c}
n-k \\
2 m
\end{array}\right)\left(\frac{1}{2}\right)_{m} \\
& \times \sum_{l=0}^{n-k-2 m}\left(\begin{array}{c}
n-k-2 m \\
l
\end{array}\right) \frac{(-1)^{l} 2^{n-k-2 m-l}(k+l+2 m+\alpha+1)_{n-k-2 m-l}}{(n+k+l+2 m+\alpha+\beta+1)_{n-k-2 m-l}} \\
= & \left(\begin{array}{l}
n \\
k
\end{array}\right) \sum_{m=0}^{[(n-k) / 2]}\left(\begin{array}{c}
n-k \\
2 m
\end{array}\right) \frac{2^{n-k-2 m}\left(\frac{1}{2}\right)_{m}(k+2 m+\alpha+1)_{n-k-2 m}}{(n+k+2 m+\alpha+\beta+1)_{n-k-2 m}} \\
& \times{ }_{2} F_{1}\left(\begin{array}{c}
2 m-n+k, n+k+2 m+\alpha+\beta+1 \mid \frac{1}{2} \\
k+2 m+\alpha+1
\end{array}\right.
\end{aligned}
$$

In the Gegenbauer case, when $\alpha=\beta$, (A.6) leads to a closed expression for $c_{n k}$,

$$
\begin{aligned}
c_{n k} & =\left(\begin{array}{l}
n \\
k
\end{array}\right) \sum_{m=0}^{[(n-k) / 2]}\left(\begin{array}{c}
n-k \\
2 m
\end{array}\right) \frac{\Gamma\left(m+\frac{1}{2}\right) \Gamma\left(\frac{n+k+1}{2}+m+\alpha\right)}{\Gamma\left(n+\alpha+\frac{1}{2}\right) \Gamma\left(\frac{k-n+1}{2}+m\right)} \\
& =\left(\begin{array}{l}
n \\
k
\end{array}\right) \frac{\Gamma\left(\frac{1}{2}\right) \Gamma\left(\frac{n+k+1}{2}+\alpha\right)}{\Gamma\left(n+\alpha+\frac{1}{2}\right) \Gamma\left(\frac{k-n+1}{2}\right)}{ }_{2} F_{0}\left(\frac{k-n}{2}, \frac{n+k+1}{2}+\alpha \mid 1\right) .
\end{aligned}
$$

We readily see that $c_{n k}$ vanishes whenever $n-k$ is odd. Therefore, writing $n-k=2 r$ with $r$ integer, the connection formula simplifies to [18, p 284]

$$
\begin{aligned}
P_{n}^{(\alpha, \alpha)}(x) & =\sum_{r=0}^{[n / 2]} c_{n r} H_{n-2 r}(x) \\
c_{n r} & =\left(\begin{array}{c}
n \\
2 r
\end{array}\right) \sum_{m=0}^{r}\left(\begin{array}{c}
2 r \\
2 m
\end{array}\right) \frac{\left(\frac{1}{2}\right)_{m}\left(\frac{1}{2}\right)_{r-m}}{\left(\frac{1}{2}-n-\alpha\right)_{r-m}} \\
& =\left(\begin{array}{c}
n \\
2 r
\end{array}\right) \frac{\left(\frac{1}{2}\right)_{r}}{\left(\frac{1}{2}-n-\alpha\right)_{r}}{ }_{2} F_{0}\left(\begin{array}{c}
-r, n-r+\alpha+\frac{1}{2} \mid \\
-
\end{array}\right) .
\end{aligned}
$$

\subsection{Expansions in series of Laguerre polynomials}

Following from (21) and (22),

$$
p_{n}(x)=\sum_{k=0}^{n} c_{n k} L_{k}^{(\alpha)}(x) \quad c_{n k}=\frac{1}{\Gamma(k+\alpha+1)}\left(\begin{array}{l}
n \\
k
\end{array}\right) \mathcal{I}_{n k}
$$

where

$$
\mathcal{I}_{n k}=\int_{0}^{\infty} p_{n-k, k}(x) x^{\alpha+k} \mathrm{e}^{-x} \mathrm{~d} x=\tilde{A}_{n-k, k} \int_{0}^{\infty} \frac{\mathrm{d}^{n-k} \tilde{\omega}_{n}(x)}{\mathrm{d} x^{n-k}} \frac{x^{\alpha+k} \mathrm{e}^{-x}}{\tilde{\omega}_{k}(x)} \mathrm{d} x .
$$


3.2.1. Connection with Hermite polynomials. For $p_{n}(x)=H_{n}(x)$, the first expression for $\mathcal{I}_{n k}$ in (36) reads

$$
\mathcal{I}_{n k}=\int_{0}^{\infty} H_{n-k}(x) x^{\alpha+k} \mathrm{e}^{-x} \mathrm{~d} x .
$$

Thus, by (A.10),

$$
\mathcal{I}_{n k}=\sum_{j=0}^{[(n-k) / 2]}\left(\begin{array}{c}
n-k \\
2 j
\end{array}\right) \frac{\left(-\frac{1}{4}\right)^{j}(2 j) ! \Gamma(n-2 j+\alpha+1)}{j !}
$$

and (35) then yields [18, p 207]

$$
\begin{aligned}
c_{n k} & =\left(\begin{array}{l}
n \\
k
\end{array}\right) \sum_{j=0}^{[(n-k) / 2]}\left(\begin{array}{c}
n-k \\
2 j
\end{array}\right)(-1)^{j}\left(\frac{1}{2}\right)_{j}(k+\alpha+1)_{n-k-2 j} \\
& =(k+\alpha+1)_{n-k}\left(\begin{array}{l}
n \\
k
\end{array}\right){ }_{2} F_{2}\left(\begin{array}{c}
\frac{k-n}{2}, \frac{k-n+1}{2} \\
\frac{-n-\alpha}{2}, \frac{-n-\alpha+1}{2}
\end{array} \mid-\frac{1}{4}\right) .
\end{aligned}
$$

3.2.2. Connection with Laguerre polynomials of different parameters. For $p_{n}(x)=L_{n}^{(\beta)}(x)$, the second expression for $\mathcal{I}_{n k}$ in (36) gives

$$
\mathcal{I}_{n k}=(-1)^{n-k} \int_{0}^{\infty} \frac{\mathrm{d}^{n-k}\left(x^{\beta+n} \mathrm{e}^{-x}\right)}{\mathrm{d} x^{n-k}} x^{\alpha-\beta} \mathrm{d} x .
$$

Integrating by parts $n-k$ times (or, equivalently, by direct use of equations (23) or (24)), we readily find that

$$
\mathcal{I}_{n k}=(-1)^{n-k} \Gamma(k+\alpha+1)(\beta-\alpha)_{n-k}
$$

and, with account of (35), one has (cf [1, p 192], [18, p 209]),

$$
c_{n k}=\left(\begin{array}{l}
n \\
k
\end{array}\right)(-1)^{n-k}(\beta-\alpha)_{n-k} .
$$

From this result, we readily see that the sign of $c_{n k}$ is $(-1)^{n-k}$ if $\beta>\alpha[30,31,33]$, while $c_{n k} \geqslant 0$ if $\beta-\alpha$ is a negative integer. Finally, if $\beta-\alpha<0$ is not an integer, $c_{n k}$ is non-negative provided that $\alpha-\beta \geqslant n-k-1$, so that all the connection coefficients are non-negative if $\alpha-\beta \geqslant n-1$.

3.2.3. Connection with Jacobi polynomials. For $p_{n}(x)=P_{n}^{(\gamma, \delta)}(x)$, the first equation in (36) gives

$$
\mathcal{I}_{n k}=\int_{0}^{\infty} P_{n-k}^{(\gamma+k, \delta+k)}(x) x^{\alpha+k} \mathrm{e}^{-x} \mathrm{~d} x
$$

Using the explicit expression of the monic Jacobi polynomials (A.12), we find that

$$
\mathcal{I}_{n k}=\sum_{j=0}^{n-k}\left(\begin{array}{c}
n-k \\
j
\end{array}\right) \frac{2^{n-k-j}(k+j+\gamma+1)_{n-k-j}}{(n+k+j+\gamma+\delta+1)_{n-k-j}} \int_{0}^{\infty}(x-1)^{j} x^{\alpha+k} \mathrm{e}^{-x} \mathrm{~d} x .
$$

Evaluating the integrals in the right-hand side, the expression for $c_{n k}$ follows in a straightforward way from (35):

$c_{n k}=\left(\begin{array}{l}n \\ k\end{array}\right) \sum_{j=0}^{n-k}\left(\begin{array}{c}n-k \\ j\end{array}\right) \frac{2^{n-k-j}(k+j+\gamma+1)_{n-k-j}}{(n+k+j+\gamma+\delta+1)_{n-k-j}} \sum_{m=0}^{j}\left(\begin{array}{c}j \\ m\end{array}\right)(-1)^{j-m}(k+\alpha+1)_{m}$. 
The sum over $m$ can be written as a ${ }_{2} F_{0}$ hypergeometric function of unit argument and upper parameters $-j, k+\alpha+1$. Interchanging the order of summation and shifting the index $j$ to $l=j-m$, we obtain the alternative expression

$$
\begin{aligned}
c_{n k}=\left(\begin{array}{l}
n \\
k
\end{array}\right) \sum_{m=0}^{n-k} & (k+\alpha+1)_{m} \sum_{l=0}^{n-k-m}\left(\begin{array}{c}
n-k \\
m+l
\end{array}\right)\left(\begin{array}{c}
m+l \\
m
\end{array}\right) \\
& \times \frac{(-1)^{l} 2^{n-k-m-l}(k+m+l+\gamma+1)_{n-k-m-l}}{(n+k+m+l+\gamma+\delta+1)_{n-k-m-l}} \\
= & \left(\begin{array}{l}
n \\
k
\end{array}\right) \sum_{m=0}^{n-k}\left(\begin{array}{c}
n-k \\
m
\end{array}\right) \frac{2^{n-k-m}(k+\alpha+1)_{m}(k+m+\gamma+1)_{n-k-m}}{(n+k+m+\gamma+\delta+1)_{n-k-m}} \\
& \times{ }_{2} F_{1}\left(\begin{array}{c|c}
m+k-n, n+k+m+\gamma+\delta+1 \\
k+m+\gamma+1
\end{array}\right) .
\end{aligned}
$$

In the Gegenbauer case, when $\gamma=\delta$, equation (A.6) leads to

$$
c_{n k}=\left(\begin{array}{l}
n \\
k
\end{array}\right) \frac{\Gamma\left(\frac{1}{2}\right)}{\Gamma\left(n+\gamma+\frac{1}{2}\right)} \sum_{m=0}^{n-k}\left(\begin{array}{c}
n-k \\
m
\end{array}\right) \frac{(k+\alpha+1)_{m} \Gamma\left(\frac{m+n+k+1}{2}+\gamma\right)}{\Gamma\left(\frac{m+k-n+1}{2}\right)} .
$$

We readily see that the $m$ th term in this summation vanishes whenever $n-k-m$ is odd. Therefore, writing $n-k-m=2 r$ with $r$ integer, the expression of the connection coefficients simplifies to

$$
\begin{aligned}
c_{n k} & =\left(\begin{array}{l}
n \\
k
\end{array}\right) \sum_{r=0}^{[(n-k) / 2]}\left(\begin{array}{c}
n-k \\
2 r
\end{array}\right) \frac{(-1)^{r}\left(\frac{1}{2}\right)_{r}(k+\alpha+1)_{n-k-2 r}}{\left(n-r+\gamma+\frac{1}{2}\right)_{r}} \\
& =(k+\alpha+1)_{n-k}\left(\begin{array}{l}
n \\
k
\end{array}\right){ }_{2} F_{3}\left(\begin{array}{c|c}
\frac{-n-\alpha}{2}, \frac{-n-\alpha+1}{2},-n-\gamma+\frac{k-n+1}{2} & \frac{1}{4}
\end{array}\right) .
\end{aligned}
$$

The particular case $\gamma=0$ of this formula, which corresponds to the expansion of Legendre polynomials in series of Laguerre polynomials, is given in [18, p 208].

\subsection{Expansions in series of Jacobi polynomials}

Following from (21) and (22),

$$
\begin{aligned}
& p_{n}(x)=\sum_{k=0}^{n} c_{n k} P_{k}^{(\alpha, \beta)}(x) \\
& c_{n k}=\frac{\Gamma(2 k+\alpha+\beta+2)}{2^{2 k+\alpha+\beta+1} \Gamma(k+\alpha+1) \Gamma(k+\beta+1)}\left(\begin{array}{l}
n \\
k
\end{array}\right) \mathcal{I}_{n k}
\end{aligned}
$$

where

$$
\begin{aligned}
\mathcal{I}_{n k} & =\int_{-1}^{1} p_{n-k, k}(x)(1-x)^{k+\alpha}(1+x)^{k+\beta} \mathrm{d} x \\
& =\tilde{A}_{n-k, k} \int_{-1}^{1} \frac{\mathrm{d}^{n-k} \tilde{\omega}_{n}(x)}{\mathrm{d} x^{n-k}} \frac{(1-x)^{k+\alpha}(1+x)^{k+\beta}}{\tilde{\omega}_{k}(x)} \mathrm{d} x .
\end{aligned}
$$

3.3.1. Connection with Hermite polynomials. For $p_{n}(x)=H_{n}(x)$, the first equation in (43) gives

$$
\mathcal{I}_{n k}=\int_{-1}^{1} H_{n-k}(x)(1-x)^{k+\alpha}(1+x)^{k+\beta} \mathrm{d} x .
$$


Substituting the explicit expression of the monic Hermite polynomials (A.10), and using (A.7),

$$
\begin{aligned}
\mathcal{I}_{n k}=\frac{(-1)^{n-k} 2^{2 k+\alpha+\beta+1} \Gamma(k+\alpha+1) \Gamma(k+\beta+1)}{\Gamma(2 k+\alpha+\beta+2)} & \\
& \times \sum_{m=0}^{[(n-k) / 2]}\left(\begin{array}{c}
n-k \\
2 m
\end{array}\right) \frac{\left(-\frac{1}{4}\right)^{m}(2 m) !}{m !}{ }_{2} F_{1}\left(\begin{array}{c}
k-n+2 m, k+\beta+1 \\
2 k+\alpha+\beta+2
\end{array}\right)
\end{aligned}
$$

so that from equation (42) we obtain

$c_{n k}=(-1)^{n-k}\left(\begin{array}{l}n \\ k\end{array}\right) \sum_{m=0}^{[(n-k) / 2]}\left(\begin{array}{c}n-k \\ 2 m\end{array}\right)(-1)^{m}\left(\frac{1}{2}\right)_{m}{ }_{2} F_{1}\left(\begin{array}{c}k-n+2 m, k+\beta+1 \\ 2 k+\alpha+\beta+2\end{array}\right)$.

In the particular case of Gegenbauer polynomials, when $\alpha=\beta$, (A.5) implies that $c_{n k}$ vanishes whenever $n-k$ is odd. Then, writing $n-k=2 r$ with $r$ integer, the connection formula simplifies to

$$
\begin{aligned}
H_{n}(x) & =\sum_{r=0}^{[n / 2]} c_{n r} P_{n-2 r}^{(\alpha, \alpha)}(x) \\
c_{n r}= & \left(\begin{array}{c}
n \\
2 r
\end{array}\right) \sum_{m=0}^{r}\left(\begin{array}{c}
2 r \\
2 m
\end{array}\right) \frac{(-1)^{m}\left(\frac{1}{2}\right)_{m}\left(\frac{1}{2}\right)_{r-m}}{\left(n-2 r+\alpha+\frac{3}{2}\right)_{r-m}} \\
= & \left(\begin{array}{c}
n \\
2 r
\end{array}\right) \frac{\left(\frac{1}{2}\right)_{r}}{\left(n-2 r+\alpha+\frac{3}{2}\right)_{r}}{ }_{2} F_{0}\left(\begin{array}{c}
\left.-r, r-n-\alpha-\frac{1}{2} \mid-1\right) .
\end{array}\right.
\end{aligned}
$$

An equivalent form of this expression is given in [18, p 284].

3.3.2. Connection with Laguerre polynomials. For $p_{n}(x)=L_{n}^{(\gamma)}(x)$, by the first equation in (43) we have

$$
\mathcal{I}_{n k}=\int_{-1}^{1} L_{n-k}^{(\gamma+k)}(x)(1-x)^{k+\alpha}(1+x)^{k+\beta} \mathrm{d} x .
$$

Then, by (A.7) and (A.11),

$$
\begin{aligned}
\mathcal{I}_{n k}=(-1)^{n-k} & 2^{2 k+\alpha+\beta+1} \frac{\Gamma(k+\alpha+1) \Gamma(k+\beta+1)}{\Gamma(2 k+\alpha+\beta+2)} \\
& \times \sum_{m=0}^{n-k}\left(\begin{array}{c}
n-k \\
m
\end{array}\right)(k+m+\gamma+1)_{n-k-m}{ }_{2} F_{1}\left(\begin{array}{c}
-m, k+\beta+1 \\
2 k+\alpha+\beta+2
\end{array}\right) .
\end{aligned}
$$

Finally, using (42), we find that

$c_{n k}=(-1)^{n-k}\left(\begin{array}{l}n \\ k\end{array}\right) \sum_{m=0}^{n-k}\left(\begin{array}{c}n-k \\ m\end{array}\right)(k+m+\gamma+1)_{n-k-m} 2 F_{1}\left(\begin{array}{c}-m, k+\beta+1 \\ 2 k+\alpha+\beta+2\end{array} \mid 2\right)$.

Again, for Gegenbauer polynomials $(\alpha=\beta$ ), equation (A.5) leads to

$$
\begin{aligned}
c_{n k}=(-1)^{n-k}\left(\begin{array}{l}
n \\
k
\end{array}\right) \sum_{m=0}^{[(n-k) / 2]}\left(\begin{array}{c}
n-k \\
2 m
\end{array}\right) \frac{\left(\frac{1}{2}\right)_{m}(k+2 m+\gamma+1)_{n-k-2 m}}{\left(k+\alpha+\frac{3}{2}\right)_{m}} \\
=(-1)^{n-k}(k+\gamma+1)_{n-k}\left(\begin{array}{l}
n \\
k
\end{array}\right){ }_{2} F_{3}\left(\begin{array}{c|c}
\frac{k-n}{2}, \frac{k-n+1}{2} \\
k+\alpha+\frac{3}{2}, \frac{k+\gamma+1}{2}, \frac{k+\gamma}{2}+1
\end{array}\right)
\end{aligned}
$$

and the sign of these coefficients is readily shown to be $(-1)^{n-k}$. The particular case $\alpha=0$ of (47), which corresponds to the expansion of Laguerre polynomials in series of Legendre polynomials, is given in [18, p 216]. 
3.3.3. Connection with Jacobi polynomials of different parameters. For $p_{n}(x)=P_{n}^{(\gamma, \delta)}(x)$, the first equation in (43) yields

$$
\mathcal{I}_{n k}=\int_{-1}^{1} P_{n-k}^{(\gamma+k, \delta+k)}(x)(1-x)^{k+\alpha}(1+x)^{k+\beta} \mathrm{d} x .
$$

Using the explicit expression of the monic Jacobi polynomials (A.12), together with (A.7), we find that

$$
\begin{aligned}
\mathcal{I}_{n k}=2^{n+k+\alpha+\beta+1} & \Gamma(k+\beta+1) \\
& \times \sum_{m=0}^{n-k}\left(\begin{array}{c}
n-k \\
m
\end{array}\right) \frac{(-1)^{m}(k+m+\gamma+1)_{n-k-m} \Gamma(k+m+\alpha+1)}{(n+k+m+\gamma+\delta+1)_{n-k-m} \Gamma(2 k+m+\alpha+\beta+2)} .
\end{aligned}
$$

Then, from (42), we obtain the connection formula

$$
\begin{aligned}
c_{n k}=\left(\begin{array}{l}
n \\
k
\end{array}\right) \sum_{m=0}^{n-k} & \left(\begin{array}{c}
n-k \\
m
\end{array}\right) \frac{(-1)^{m} 2^{n-k}(k+\alpha+1)_{m}(k+m+\gamma+1)_{n-k-m}}{(2 k+\alpha+\beta+2)_{m}(n+k+m+\gamma+\delta+1)_{n-k-m}} \\
= & \frac{2^{n-k}(k+\gamma+1)_{n-k}}{(n+k+\gamma+\delta+1)_{n-k}}\left(\begin{array}{l}
n \\
k
\end{array}\right) \\
& \times{ }_{3} F_{2}\left(\begin{array}{c}
k-n, n+k+\gamma+\delta+1, k+\alpha+1 \\
k+\gamma+1,2 k+\alpha+\beta+2
\end{array}\right)
\end{aligned}
$$

which, according to Askey [12, 29], was first derived by Feldheim [36]. A complete discussion of the non-negativity cases of these coefficients can be found in [32] (see also $[29,30,33,34])$. As pointed out by Askey [12], there are three important particular cases when the hypergeometric function in (48) can be evaluated in closed form by use of standard summation formulae.

For Gegenbauer polynomials, when $\alpha=\beta$ and $\gamma=\delta$, the classical Watson summation theorem (A.8) leads to

$$
c_{n k}=\left(\begin{array}{l}
n \\
k
\end{array}\right) \frac{\Gamma\left(k+\alpha+\frac{3}{2}\right) \Gamma\left(\frac{n+k+1}{2}+\gamma\right) \Gamma\left(\frac{1}{2}\right) \Gamma(\alpha-\gamma+1)}{\Gamma\left(n+\gamma+\frac{1}{2}\right) \Gamma\left(\frac{n+k+3}{2}+\alpha\right) \Gamma\left(\frac{k-n+1}{2}\right) \Gamma\left(\frac{k-n}{2}+\alpha-\gamma+1\right)} .
$$

We readily see that $c_{n k}$ vanishes whenever $n-k$ is odd. Therefore, writing $n-k=2 r$ with $r$ integer, the connection formula simplifies to

$$
\begin{aligned}
& P_{n}^{(\gamma, \gamma)}(x)=\sum_{r=0}^{[n / 2]} c_{n r} P_{n-2 r}^{(\alpha, \alpha)}(x) \\
& c_{n r}=\left(\begin{array}{c}
n \\
2 r
\end{array}\right) \frac{\left(\frac{1}{2}\right)_{r}(\gamma-\alpha)_{r}}{\left(n-r+\gamma+\frac{1}{2}\right)_{r}\left(n-2 r+\alpha+\frac{3}{2}\right)_{r}} .
\end{aligned}
$$

Now we find that $c_{n r}>0$ if $\gamma>\alpha$ [12], while the sign of $c_{n r}$ is $(-1)^{r}$ if $\alpha-\gamma>r-1$, so that all the connection coefficients have sign $(-1)^{r}$ if $\alpha-\gamma>[n / 2]-1$.

On the other hand, in the particular case when $\alpha=\gamma$, the ${ }_{3} F_{2}$ hypergeometric function in (48) reduces to a ${ }_{2} F_{1}$ function of unit argument, which can be evaluated in closed form by means of the Chu-Vandermonde theorem (A.4). We thus obtain,

$$
c_{n k}=\left(\begin{array}{l}
n \\
k
\end{array}\right) \frac{2^{n-k}(k+\alpha+1)_{n-k}(k-n+\beta-\delta+1)_{n-k}}{(n+k+\alpha+\delta+1)_{n-k}(2 k+\alpha+\beta+2)_{n-k}} .
$$

These coefficients have sign $(-1)^{n-k}$ if $\delta>\beta$, while they are non-negative if $\beta-\delta$ is a positive integer [12]. If $\beta-\delta>0$ is not an integer, then the connection coefficients are non-negative provided that $\beta-\delta \geqslant n-k-1$, so that all of them are non-negative if $\beta-\delta \geqslant n-1$. 
A similar simplification of (48) can be achieved when $\beta=\delta$ by means of the PfaffSaalschütz formula (A.9), which leads to

$$
c_{n k}=\left(\begin{array}{l}
n \\
k
\end{array}\right) \frac{(-2)^{n-k}(k+\beta+1)_{n-k}(k-n+\alpha-\gamma+1)_{n-k}}{(n+k+\beta+\gamma+1)_{n-k}(2 k+\alpha+\beta+2)_{n-k}} .
$$

In fact, this case turns out to be equivalent to the previous one because of the symmetry relation $P_{n}^{(\alpha, \beta)}(-x)=(-1)^{n} P_{n}^{(\beta, \alpha)}(x)$, and the same happens for the sign of the connection coefficients. Now, they are positive if $\gamma>\alpha[3,12,31,33]$, while their sign is $(-1)^{n-k}$ if $\alpha-\gamma>n-k-1$, so that all of them have sign $(-1)^{n-k}$ if $\alpha-\gamma>n-1$.

\section{Some examples of generalized linearization formulae}

There are 18 different linearization formulae of the form (2) involving the three classical families, which correspond to the expansion of the six possible products in series of each family. Since we have just computed the complete set of connection coefficients for these polynomials, the generalized linearization coefficients can be conveniently evaluated by means of (28). We shall illustrate how this formula works by means of some examples.

\subsection{Expansions of products involving a Hermite polynomial in series of Hermite polynomials}

We look for the coefficients of

$$
p_{n}(x) H_{m}(x)=\sum_{k=0}^{n+m} g_{n m k} H_{k}(x)
$$

where $H_{m}(x)$ denotes the monic Hermite polynomial of degree $m$. In this case, we trivially have $c_{m+j-k, r}^{(k-j, k)}(q)=\delta_{m+j-k, r}$, so that (28) reduces to

$$
\begin{aligned}
g_{n m k} & =\frac{1}{\gamma_{k}} \sum_{j=j_{-}}^{j_{+}}\left(\begin{array}{l}
n \\
j
\end{array}\right)\left(\begin{array}{c}
m \\
k-j
\end{array}\right) c_{n-j, m+j-k}^{(j, k)}(p) \varkappa_{m+j-k, k} \\
& =2^{k-m} \sum_{j=j_{-}}^{j_{+}}\left(\begin{array}{l}
n \\
j
\end{array}\right)\left(\begin{array}{c}
m \\
k-j
\end{array}\right) \frac{(m+j-k) !}{2^{j}} c_{n-j, m+j-k}^{(j, k)}(p) .
\end{aligned}
$$

If $p_{n}(x)=H_{n}(x)$, then we also have $c_{n-j, m+j-k}^{(j, k)}(p)=\delta_{n-j, m+j-k}$. From (53) we readily see that, in the (standard) linearization formula for Hermite polynomials,

$$
H_{n}(x) H_{m}(x)=\sum_{k=|n-m|}^{n+m} l_{n m k} H_{k}(x)
$$

the coefficient $l_{n m k}$ vanishes whenever $n+m-k$ is odd, so that the sum over $k$ in (54) can be restricted to the values $k=n+m-2 r$ with integer $r$. For such values of $k$, the only non-vanishing term in the summation over $j$ in the expression for $l_{n m k}$ given by (53) is that corresponding to $j=n-r$, so that we have for $k=n+m-2 r$,

$$
l_{n m k}=\left(\begin{array}{c}
n \\
r
\end{array}\right)\left(\begin{array}{c}
m \\
r
\end{array}\right) \frac{r !}{2^{r}} .
$$

Thus we obtain the well known Feldheim formula (cf [37], [1, p 195]),

$$
H_{n}(x) H_{m}(x)=\sum_{r=0}^{\min (n, m)}\left(\begin{array}{l}
n \\
r
\end{array}\right)\left(\begin{array}{l}
m \\
r
\end{array}\right) \frac{r !}{2^{r}} H_{n+m-2 r}(x) .
$$


The linearization coefficients in this formula are obviously positive, which has been found useful in applications [38].

If $p_{n}(x)=L_{n}^{(\alpha)}(x)$, the connection coefficients $c_{n-j, m+j-k}^{(j, k)}(p)$ in (53) correspond to the expansion

$$
L_{n-j}^{(\alpha+j)}(x)=\sum_{r=0}^{n-j} c_{n-j, r}^{(j, k)}(p) H_{r}(x)
$$

and the expression for these coefficients derived from (31) leads to

$$
\begin{gathered}
g_{n m k}=(-1)^{n-m+k} 2^{k-m} \sum_{j=j_{-}}^{j_{+}}\left(\begin{array}{c}
n \\
j
\end{array}\right)\left(\begin{array}{c}
m \\
k-j
\end{array}\right)\left(\begin{array}{c}
n-j \\
m+j-k
\end{array}\right) \frac{(m+j-k) !(n+\alpha+1)_{m-n-k+2 j}}{2^{j}} \\
\times{ }_{2} F_{2}\left(\begin{array}{c}
\frac{m-n-k}{2}+j, \frac{m-n-k+1}{2}+j \\
\frac{m-k+\alpha+1}{2}+j, \frac{1}{2}+j+1
\end{array}\right) .
\end{gathered}
$$

We readily see that the sign of these coefficients is $(-1)^{n-m+k}$. Likewise, when $p_{n}(x)=$ $P_{n}^{(\alpha, \beta)}(x)$, using equations (32) and (33) we can write $g_{n m k}$ as a double sum of terminating ${ }_{2} F_{0}$ or ${ }_{2} F_{1}$ hypergeometric functions, which in the Gegenbauer case $(\alpha=\beta)$ reduce to a simple sum of ${ }_{2} F_{0}$ functions (cf (34)).

\subsection{Linearization formulae for Laguerre polynomials}

Let us consider now the linearization problem

$$
L_{n}^{(\alpha)}(x) L_{m}^{(\beta)}(x)=\sum_{k=0}^{n+m} g_{n m k} L_{k}^{(\gamma)}(x)
$$

where $L_{n}^{(\alpha)}(x)$ denotes the monic Laguerre polynomial of degree $n$ and parameter $\alpha$; according to (12), the linearization coefficients $g_{n m k}$ have the integral representation

$$
g_{n m k}=\frac{1}{\Gamma(k+\gamma+1) k !} \int_{0}^{\infty} L_{n}^{(\alpha)}(x) L_{m}^{(\beta)}(x) L_{k}^{(\gamma)}(x) x^{\gamma} \mathrm{e}^{-x} \mathrm{~d} x .
$$

Equation (28) now reads

$$
\begin{aligned}
& g_{n m k}=\frac{1}{\Gamma(k+\gamma+1)} \sum_{j=j_{-}}^{j_{+}}\left(\begin{array}{c}
n \\
j
\end{array}\right)\left(\begin{array}{c}
m \\
k-j
\end{array}\right) \mathcal{I}_{n m k}(j) \\
& \mathcal{I}_{n m k}(j)=\sum_{r=0}^{r_{+}} c_{n-j, r}^{(j, k)} c_{m+j-k, r}^{(k-j, k)} \Gamma(r+k+\gamma+1) r !
\end{aligned}
$$

where the connection coefficients correspond to the expansions

$$
L_{n-j}^{(\alpha+j)}(x)=\sum_{r=0}^{n-j} c_{n-j, r}^{(j, k)} L_{r}^{(\gamma+k)}(x) \quad L_{m+j-k}^{(\beta+k-j)}(x)=\sum_{r=0}^{m+j-k} c_{m+j-k, r}^{(k-j, k)} L_{r}^{(\gamma+k)}(x) .
$$

From (38), we find that the explicit form of these coefficients is

$$
\begin{aligned}
& c_{n-j, r}^{(j, k)}=\left(\begin{array}{c}
n-j \\
r
\end{array}\right)(-1)^{n-j-r}(\alpha-\gamma+j-k)_{n-j-r} \\
& c_{m+j-k, r}^{(k-j, k)}=\left(\begin{array}{c}
m+j-k \\
r
\end{array}\right)(-1)^{m+j-k-r}(\beta-\gamma-j)_{m+j-k-r} .
\end{aligned}
$$


Substituting these expressions into (58), we obtain

$$
\begin{aligned}
g_{n m k}=\sum_{j=j_{-}}^{j_{+}}\left(\begin{array}{c}
n \\
j
\end{array}\right)\left(\begin{array}{c}
m \\
k-j
\end{array}\right) \sum_{r=0}^{r_{+}}\left(\begin{array}{c}
n-j \\
r
\end{array}\right)\left(\begin{array}{c}
m+j-k \\
r
\end{array}\right) r !(k+\gamma+1)_{r} \\
\\
\quad \times(\gamma-\alpha+k-n+r+1)_{n-j-r}(\gamma-\beta+k-m+r+1)_{m+j-k-r} .
\end{aligned}
$$

We readily see that, if $\gamma-\alpha$ and $\gamma-\beta$ are non-negative integers, then $g_{n m k} \geqslant 0$. This result generalizes the non-negativity condition obtained by Koornwinder [34] for the particular case when $\alpha$ and $\beta$ are integers and $\gamma=\alpha+\beta$. We also see from (59) that, if $\gamma-\alpha$ (resp. $\gamma-\beta$ ) is not an integer, the non-negativity of the linearization coefficients still holds provided that $\gamma-\alpha>n-1$ (resp. $\gamma-\beta>m-1$ ). On the other hand, if $\alpha-\gamma>k-j_{-}$and $\beta-\gamma>j_{+}$, then the sign of $g_{n m k}$ is $(-1)^{n+m-k}$, so that all the linearization coefficients have this sign if $\alpha-\gamma>m$ and $\beta-\gamma>n$.

The summation over $r$ in (59) can be expressed as a ${ }_{3} F_{2}$ hypergeometric function of unit argument, which leads to

$$
\begin{gathered}
g_{n m k}=\sum_{j=j_{-}}^{j_{+}}\left(\begin{array}{c}
n \\
j
\end{array}\right)\left(\begin{array}{c}
m \\
k-j
\end{array}\right)(\gamma-\alpha+k-n+1)_{n-j}(\gamma-\beta+k-m+1)_{m+j-k} \\
\quad \times{ }_{3} F_{2}\left(\begin{array}{c}
j-n, k-j-m, k+\gamma+1 \\
\gamma-\alpha+k-n+1, \gamma-\beta+k-m+1
\end{array} \mid 1\right) .
\end{gathered}
$$

In the particular case when $\gamma=\alpha+\beta$, the expression for the linearization coefficients given by the previous formula can be further simplified by taking advantage of the Pfaff-Saalschütz summation theorem (A.9), which yields

$$
\begin{aligned}
g_{n m k}=\sum_{j=j_{-}}^{j_{+}}\left(\begin{array}{l}
n \\
j
\end{array}\right)\left(\begin{array}{c}
m \\
k-j
\end{array}\right)(k-m+n-j+\alpha+1)_{m+j-k}(m-n+j+\beta+1)_{n-j} \\
=\left(\begin{array}{c}
m \\
k
\end{array}\right)(n-m+k+\alpha+1)_{m-k}(m-n+\beta+1)_{n} \\
\\
\times{ }_{3} F_{2}\left(\begin{array}{c}
-n,-k, m-n-k-\alpha \\
m-k+1, m-n+\beta+1
\end{array} \mid-1\right) .
\end{aligned}
$$

We already know, from the discussion after (59), that $g_{n m k} \geqslant 0$ if $\alpha, \beta \in \mathbb{Z}$, while if $\alpha$ (resp. $\beta$ ) is not an integer the linearization coefficients are still non-negative provided that $\alpha>m-1$ (resp. $\beta>n-1$ ). Equation (61) enables us to improve on the latter result, since inspection of its right-hand side shows that, if $\alpha$ (resp. $\beta$ ) is not an integer, the non-negativity of the linearization coefficients holds under a less restrictive condition, namely that $\alpha>m-n-1$ (resp. $\beta>n-m-1$ ); in particular, the coefficients are non-negative in the $m=n$ case.

Now let us turn to (59). Interchanging the order of summation and shifting the index $j$ to $l=n-r-j$, this formula can be written as

$$
\begin{aligned}
g_{n m k}=m ! \sum_{r=0}^{r_{+}} & \left(\begin{array}{l}
n \\
r
\end{array}\right) \frac{(k+\gamma+1)_{r}(\gamma-\beta+k-m+r+1)_{m+n-k-2 r}}{(m+n-k-2 r) ! \Gamma(k-n+r+1)} \\
& \times{ }_{3} F_{2}\left(\begin{array}{c}
r-n, k-m-n+2 r, \gamma-\alpha+k-n+r+1 \\
\beta-\gamma-n+r, k-n+r+1
\end{array} \mid-1\right) .
\end{aligned}
$$

In the particular case when $\alpha=\beta=\gamma$, the ${ }_{3} F_{2}$ hypergeometric function in the right-hand side of this equation reduces to a ${ }_{1} F_{0}$ one, which can be evaluated in closed form by means 
of (A.3). Thus we find that the solution of the standard linearization problem for Laguerre polynomials,

$$
L_{n}^{(\alpha)}(x) L_{m}^{(\alpha)}(x)=\sum_{k=|n-m|}^{n+m} l_{n m k} L_{k}^{(\alpha)}(x)
$$

is given by

$$
\begin{aligned}
l_{n m k}=m ! \sum_{r=0}^{r_{+}} & \left(\begin{array}{l}
n \\
r
\end{array}\right) \frac{2^{m+n-k-2 r}(k+\alpha+1)_{r}(k-m+r+1)_{m+n-k-2 r}}{(m+n-k-2 r) ! \Gamma(k-n+r+1)} \\
= & \frac{2^{m+n-k} n ! m !}{(m+n-k) ! \Gamma(k-n+1) \Gamma(k-m+1)} \\
& \times{ }_{3} F_{2}\left(\begin{array}{c|c}
\frac{k-m-n}{2}, \frac{k-m-n+1}{2}, k+\alpha+1 \\
k-n+1, k-m+1
\end{array}\right) .
\end{aligned}
$$

These coefficients are non-negative, as follows from the discussion after (59); this result is a particular case of a rather general non-negativity theorem for integrals of products of Laguerre polynomials of the same parameter, which is related to the combinatorial interpretation of these integrals $[12$, lecture six]. It is worth noting that, while alternative expressions for the Laguerre linearization coefficients in the cases $\gamma=\alpha+\beta$ and $\alpha=\beta=\gamma$ can be found in the literature [16,37], the remarkably compact expressions (61) and (64) appear to be new. Let us also note that the hypergeometric function in (64) can be evaluated in closed form by means of the Pfaff-Saalschütz formula (A.9) in the case $\alpha=-\frac{1}{2}$.

\section{Conclusions}

In this work, we have described a method to solve the general hypergeometric linearization problem, i.e. the expansion of products of two arbitrary continuous hypergeometric-type polynomials in terms of a sequence of orthogonal hypergeometric polynomials. Our approach allows us to find integral representations for the associated linearization and connection coefficients, in terms of the coefficients of the differential operators corresponding to the involved polynomials, which are suitable for symbolic manipulation. To illustrate the method, we have found the explicit expressions of connection and linearization coefficients for the three classical families with real orthogonality (Hermite, Laguerre and Jacobi). These coefficients are generally given in the form of terminating hypergeometric series, which at times can be evaluated in closed form by means of classical summation theorems. In several cases, we have been able to obtain sign properties such as positivity or non-negativity conditions from the explicit representations found for the coefficients.

It is worth noting that an affine transformation of the variable preserves the hypergeometric character of the polynomial families, so that our method is also applicable in these cases. Furthermore, the present approach can be extended straightforwardly to hypergeometric polynomials in a discrete variable [39], as well as to $q$-polynomials [40]. It is complementary to the recursive approach [15], which supplies the linearization coefficients recurrently but makes use of two or more characterization properties of the involved polynomials.

In our opinion, our method is a good starting point on the long road to solving the general problem of linearization of products of arbitrary special functions other than hypergeometric-type polynomials. Particular cases of this general problem corresponding to Bessel functions, Whittaker functions, Jacobi functions, spheroidal wavefunctions and some associated hypergeometric polynomials have been recently considered [28,41]. Some further steps on the aforesaid road are the following, as yet unsolved, problems: the linearization 
of basis-set functions, the expansion of products of special functions in terms of orthogonal hypergeometric-type polynomials, the expansion of arbitrary special functions in terms of products of two hypergeometric-type polynomials [42], the linearization of products of two Nikiforov-Uvarov functions [5], the linearization and connection of two associated hypergeometric-type polynomials, the determination of generating functions of products of two hypergeometric-type functions [43], and the study of linear dependences among products of basis-set functions [44]. Solutions to these problems would give us profound insight into the algebraic properties of the special functions themselves, which would be very useful in other branches of mathematics and applied science since, in particular, it would allow us to gain insight into the matrix elements of the observables characterizing quantum mechanical systems.

\section{Acknowledgments}

This work was partially supported by research grants from the Dirección General de Enseñanza Superior (DGES) of Spain (project codes PB95-1205 for AMF and JSD, and PB96-0120 for JSR), the European Union (INTAS-93-219-ext, for JSR, AMF and JSD), and the Junta de Andalucía (FQM0207 for JSR and JSD, FQM0229 for PLA and AMF).

\section{Appendix A. Notations and formulae}

\section{A.1. Some special functions}

We use the standard notations for the Gamma function, binomial coefficients and the Pochhammer symbol, as well as their well known identities

$$
\begin{aligned}
(x)_{n} & =x(x+1) \ldots(x+n-1)=\frac{\Gamma(x+n)}{\Gamma(x)}=\frac{(-1)^{n} \Gamma(1-x)}{\Gamma(1-x-n)} \\
\left(\begin{array}{l}
z \\
k
\end{array}\right) & =\frac{(-1)^{k}(-z)_{k}}{k !} \quad \Gamma(2 z)=\frac{2^{2 z-1} \Gamma\left(z+\frac{1}{2}\right) \Gamma(z)}{\Gamma\left(\frac{1}{2}\right)} .
\end{aligned}
$$

Assuming $n$ to be a non-negative integer, we readily see from the definition of the Pochhammer symbol that $(x)_{n}>0$ if $x>0$, while the sign of $(x)_{n}$ is $(-1)^{n}$ for $x<1-n$. On the other hand, if $1-n \leqslant x \leqslant 0,(x)_{n}=0$ if $x \in \mathbb{Z}$, while otherwise its sign is $(-1)^{[1-x]}$.

The generalized hypergeometric function ${ }_{p} F_{q}$ is defined as

$$
{ }_{p} F_{q}\left(\begin{array}{c}
a_{1}, a_{2}, \ldots, a_{p} \\
b_{1}, b_{2}, \ldots, b_{q}
\end{array} \mid x\right)=\sum_{k=0}^{\infty} \frac{\left(a_{1}\right)_{k}\left(a_{2}\right)_{k} \ldots\left(a_{p}\right)_{k}}{\left(b_{1}\right)_{k}\left(b_{2}\right)_{k} \ldots\left(b_{q}\right)_{k}} \frac{x^{k}}{k !} .
$$

In the simple case when $p=1, q=0$, Newton's binomial theorem states that

$$
{ }_{1} F_{0}\left(\begin{array}{c}
a \\
-\mid
\end{array}\right)=(1-x)^{-a} \text {. }
$$

For the Gauss hypergeometric function $(p=2, q=1)$ we have the special values (see, e.g., [18]),

$$
{ }_{2} F_{1}\left(\begin{array}{c|c}
-n, b & 1 \\
c & 1
\end{array}\right)=\frac{(c-b)_{n}}{(c)_{n}}
$$

(Chu-Vandermonde sum),

$$
{ }_{2} F_{1}\left(\begin{array}{c}
-n, c \\
2 c
\end{array} \mid 2\right)= \begin{cases}0 & \text { if } n \text { is odd } \\
\frac{\left(\frac{1}{2}\right)_{n / 2}}{\left(c+\frac{1}{2}\right)_{n / 2}} & \text { if } n \text { is even }\end{cases}
$$


and

$$
{ }_{2} F_{1}\left(\begin{array}{c}
a, b \\
\frac{a+b+1}{2}
\end{array} \mid \frac{1}{2}\right)=\frac{\Gamma\left(\frac{1}{2}\right) \Gamma\left(\frac{a+b+1}{2}\right)}{\Gamma\left(\frac{a+1}{2}\right) \Gamma\left(\frac{b+1}{2}\right)} .
$$

We also have the useful integration formula (see, e.g., [18, p 69]),

$$
\begin{aligned}
\int_{a}^{b}(x-a)^{\mu-1}(b-x)^{\nu-1}(c x+d)^{\gamma} \mathrm{d} x & \\
=(b-a)^{\mu+\nu-1}(a c+d)^{\gamma} & \frac{\Gamma(\mu) \Gamma(v)}{\Gamma(\mu+\nu)}{ }_{2} F_{1}\left(\begin{array}{c}
-\gamma, \mu \\
\mu+v
\end{array} \mid \frac{c(a-b)}{a c+d}\right) .
\end{aligned}
$$

Finally, two important results concerning the ${ }_{3} F_{2}$ hypergeometric function are the classical Watson's summation theorem (see, e.g., [1, section 4.4] or [4, section 5.2.4]),

$$
{ }_{3} F_{2}\left(\begin{array}{c}
a, b, c \\
\frac{a+b+1}{2}, 2 c
\end{array} \mid 1\right)=\frac{\Gamma\left(\frac{1}{2}\right) \Gamma\left(c+\frac{1}{2}\right) \Gamma\left(\frac{a+b+1}{2}\right) \Gamma\left(\frac{1-a-b}{2}+c\right)}{\Gamma\left(\frac{a+1}{2}\right) \Gamma\left(\frac{b+1}{2}\right) \Gamma\left(\frac{1-a}{2}+c\right) \Gamma\left(\frac{1-b}{2}+c\right)}
$$

and the Pfaff-Saalschütz formula (see, e.g., [1, p 66]),

$$
{ }_{3} F_{2}\left(\begin{array}{c}
a, b,-n \\
d, a+b-n-d+1
\end{array} \mid 1\right)=\frac{(d-a)_{n}(d-b)_{n}}{(d)_{n}(d-a-b)_{n}} .
$$

The previous summation formulae hold whenever the hypergeometric series in the left-hand side are either terminating (here we always assume the parameter $n$ to be a non-negative integer) or convergent; a detailed account of the validity conditions of each theorem can be found in the indicated references.

\section{A.2. Classical hypergeometric polynomials}

We deal with the three classical families of monic hypergeometric polynomials orthogonal on the real axis: Hermite, Laguerre and Jacobi, with their standard notation. In particular, we use the following explicit formulae (see, e.g., [45]):

- Hermite polynomials:

$$
H_{n}(x)=\sum_{k=0}^{[n / 2]}\left(\begin{array}{c}
n \\
2 k
\end{array}\right) \frac{\left(-\frac{1}{4}\right)^{k}(2 k) !}{k !} x^{n-2 k}=x_{2}^{n} F_{0}\left(\begin{array}{c}
\frac{-n}{2}, \frac{1-n}{2} \\
-
\end{array} \mid-\frac{1}{x^{2}}\right) .
$$

- Laguerre polynomials:

$$
\begin{aligned}
L_{n}^{(\alpha)}(x) & =(-1)^{n} \sum_{k=0}^{n}\left(\begin{array}{l}
n \\
k
\end{array}\right)(k+\alpha+1)_{n-k}(-x)^{k} \\
& =(-1)^{n}(\alpha+1)_{n 1} F_{1}\left(\begin{array}{c}
-n \\
\alpha+1
\end{array} \mid x\right) \quad \alpha>-1 .
\end{aligned}
$$

- Jacobi polynomials:

$$
\begin{aligned}
P_{n}^{(\alpha, \beta)}(x)= & \sum_{k=0}^{n}\left(\begin{array}{l}
n \\
k
\end{array}\right) \frac{2^{n-k}(k+\alpha+1)_{n-k}}{(n+k+\alpha+\beta+1)_{n-k}}(x-1)^{k} \\
& =\frac{2^{n}(\alpha+1)_{n}}{(n+\alpha+\beta+1)_{n}}{ }_{2} F_{1}\left(\begin{array}{c}
-n, n+\alpha+\beta+1 \\
\alpha+1
\end{array} \mid \frac{1-x}{2}\right) \quad \alpha, \beta>-1 .
\end{aligned}
$$

In the particular case when $\alpha=\beta$, Jacobi polynomials are called Gegenbauer or ultraspherical polynomials. In turn, some especially important particular cases of 
Table A1. General data of the three classical families of monic orthogonal polynomials on the real axis.

\begin{tabular}{llll}
\hline$p_{n}(x)$ & $H_{n}(x)$ & $L_{n}^{(\alpha)}(x)$ & $P_{n}^{(\alpha, \beta)}(x)$ \\
\hline$(a, b)$ & $(-\infty, \infty)$ & $(0, \infty)$ & $(-1,1)$ \\
$\sigma(x)$ & 1 & $x$ & $1-x^{2}$ \\
$\tau(x)$ & $-2 x$ & $\alpha+1-x$ & $\beta-\alpha-(\alpha+\beta+2) x$ \\
$\omega_{k}(x)$ & $\mathrm{e}^{-x^{2}}$ & $x^{\alpha+k} \mathrm{e}^{-x}$ & $(1-x)^{\alpha+k}(1+x)^{\beta+k}$ \\
$\gamma_{k}$ & $\sqrt{\pi}$ & $\Gamma(k+\alpha+1)$ & $\frac{2^{2 k+\alpha+\beta+1} \Gamma(k+\alpha+1) \Gamma(k+\beta+1)}{\Gamma(2 k+\alpha+\beta+2)}$ \\
$A_{n, k}$ & $(-2)^{-n}$ & $(-1)^{n}$ & $\frac{(-1)^{n}}{(n+2 k+\alpha+\beta+1)_{n}}$ \\
$p_{n, k}(x)$ & $H_{n}(x)$ & $L_{n}^{(\alpha+k)}(x)$ & $P_{n}^{(\alpha+k, \beta+k)}(x)$ \\
\hline
\end{tabular}

Gegenbauer polynomials are the Legendre polynomials $(\alpha=\beta=0)$, Chebyshev polynomials of the first kind $\left(\alpha=\beta=-\frac{1}{2}\right)$, and Chebyshev polynomials of the second kind $\left(\alpha=\beta=\frac{1}{2}\right)$.

All the necessary data concerning these families of polynomials (see, e.g., [1]) are gathered in table A1.

\section{References}

[1] Erdélyi A 1953 (ed) Higher Transcendental Functions vol II (New York: McGraw-Hill)

[2] Erdélyi A 1954 (ed) Tables of Integral Transforms vols I-II (New York: McGraw-Hill)

[3] Szegö G 1959 Orthogonal Polynomials (Am. Math. Soc. Coll. Publ. vol 23) (Providence, RI: American Mathematical Society)

[4] Luke Y L 1975 Mathematical Functions and their Approximations (New York: Academic)

[5] Nikiforov A F and Uvarov V B 1988 Special Functions in Mathematical Physics (Basel: Birkhaüser)

[6] Lesky P 1991 Orthogonal polynomials and eigenvalue problems, unpublished

Lesky P 1996 Finite and infinite systems of continuous classical orthogonal polynomials Z. Angew. Math. Mech. 76 181-4 (in German)

[7] Bagrov V G and Gitman D M 1990 Exact Solutions of Relativistic Wave Equations (Dordrecht: Kluwer)

[8] Dehesa J S, Dominguez Adame F, Arriola E R and Zarzo A 1991 Hydrogen atom and orthogonal polynomials Orthogonal Polynomials and their Applications ed C Brezinski et al (Geneva: Baltzer) pp 223-9

[9] Khare A and Bhaduri R K 1994 Exactly solvable non-central potentials in two and three dimensions Am. J. Phys. 62 1008-14

Znojil M 1996 Jacobi polynomials and bound states J. Math. Chem. 19 205-13

[10] Jia C S, Wang X G, Yao X K, Chen P C and Xiao W 1998 A unified recurrence operator method for obtaining normalized explicit wavefunctions for shape-invariant potentials J. Phys. A Math. Gen. 31 4763-72

[11] Hobson E W 1968 The Theory of Spherical and Ellipsoidal Harmonics (New York: Chelsea) Avery J 1989 Hyperspherical Harmonics, Applications to Quantum Theory (Dordrecht: Kluwer)

[12] Askey R 1975 Orthogonal Polynomials and Special Functions (Regional Conf. Series in Appl. Math. vol 21) (Philadelphia, PA: SIAM)

[13] Budzinski J 1992 Evaluation of two-centre, two- and three-electron integrals involving correlation factors over Slater-type orbitals: II. Kinetic and potential energy integrals and examples of numerical results Int. J. Quantum Chem. 41 359-70

[14] Vilenkin N Ja and Klymik A U 1993 Representations of Lie Groups and Special Functions vol II (Dordrecht: Kluwer)

[15] Ronveaux A, Area I, Godoy E and Zarzo A 1997 Lectures on recursive approach to connection and linearization coefficients between polynomials Special Functions and Differential Equations ed K Srinivasa Rao et al (New Delhi: Allied)

Lewanowicz S 1998 A general approach to the connection and linearization problems for the classical orthogonal polynomials Preprint

[16] Niukkanen A W 1985 Clebsch-Gordan-type linearization for the products of Laguerre polynomials and hydrogen-like functions J. Phys. A Math. Gen. 18 1399-417 
[17] Edmonds A R M 1957 Angular Momentum in Quantum Mechanics (Princeton, NJ: Princeton University Press)

[18] Rainville E D 1960 Special Functions (New York: Macmillan)

[19] Rahman M 1981 A non-negative representation of the linearization coefficients of the product of Jacobi polynomials Can. J. Math. 33 915-28

Lewanowicz S 1998 The hypergeometric functions approach to the connection problem for the classical orthogonal polynomials Preprint

[20] Markett C 1994 Linearization of the product of symmetric orthogonal polynomials Constr. Approx. 10 317-38

[21] Ronveaux A, Zarzo A and Godoy E 1995 Recurrence relation for connection coefficients between two families of orthogonal polynomials J. Comput. Appl. Math. 62 67-73

Lewanowicz S 1996 Second-order recurrence relation for the linearization coefficients of the classical orthogonal polynomials J. Comput. Appl. Math. 69 159-70

Godoy E, Ronveaux A, Zarzo A and Area I 1997 Minimal recurrence relations for connection coefficients between classical orthogonal polynomials: the continuous case J. Comput. Appl. Math. 84 257-75

[22] Kibler M, Ronveaux A and Negadi T 1986 On the hydrogen-oscillator connection: passage formulae between wavefunctions J. Math. Phys. 27 1541-8

[23] Kleinsdienst H and Lüchow A 1993 Multiplication theorems for orthogonal polynomials Int. J. Quantum Chem. $48239-47$

[24] Kostelecky V A and Russell N 1996 Radial Coulomb and oscillator systems in arbitrary dimensions J. Math. Phys. 37 2166-81

[25] Shlomo S 1983 Sum rules for harmonic oscillator brackets J. Phys. A Math. Gen. 16 3463-9

[26] Sánchez-Ruiz J and Dehesa J S 1998 Expansions in series of orthogonal hypergeometric polynomials J. Comput. Appl. Math. 89 155-70

Artés P L, Dehesa J S, Martínez-Finkelshtein A and Sánchez-Ruiz J 1998 Linearization and connection coefficients for hypergeometric-type polynomials J. Comput. Appl. Math. 99 15-26

[27] Connett W C, Markett C and Schwartz A L 1992 Convolution and hypergroup structures associated with a class of Sturm-Liouville structures Trans. Am. Math. Soc. 332 365-90

Connett W C, Markett C and Schwartz A L 1992 Jacobi polynomials and related hypergroup structures Probability Measures on Groups ed H Heyer (New York: Plenum) pp 45-81

[28] Markett C 1985 Produktformeln für Eigenfunktionen singularér Sturm-Liouville Gleichungen und verallgemeinerte Translationoperationen Habilitationsschrift RWTH Aachen

[29] Askey R 1968 Jacobi polynomial expansions with positive coefficients and imbeddings of projective spaces Bull. Am. Math. Soc. 74 301-4

[30] Gasper G 1975 Positivity and special functions Theory and Application of Special Functions ed R A Askey (New York: Academic) pp 375-433

[31] Askey R 1965 Orthogonal expansions with positive coefficients Proc. Am. Math. Soc. 26 1191-4

[32] Askey R and Gasper G 1971 Jacobi polynomial expansions of Jacobi polynomials with non-negative coefficients Proc. Camb. Phil. Soc. 70 243-55

[33] Trench W F 1976 Orthogonal polynomial expansions with non-negative coefficients SIAM J. Math. Anal. 7 824-33

[34] Koornwinder T 1978 Positivity proofs for linearization and connection coefficients of orthogonal polynomials satisfying an addition formula J. London Math. Soc. 18 101-14

[35] Krattenthaler C 1995 HYP and HYPQ. Mathematica packages for the manipulation of binomial sums and hypergeometric series, respectively $q$-binomial sums and basic hypergeometric series $J$. Symb. Comput. 20 737-44

[36] Feldheim E 1941 Contributions à la théorie des polynomes de Jacobi Mat. Fiz. Lapok 48 453-504 (in Hungarian, French summary)

[37] Feldheim E 1938 Quelques nouvelles relations pour les polynomes d'Hermite J. London Math. Soc. 13 22-9 Watson G N 1938 A note on the polynomials of Hermite and Laguerre J. London Math. Soc. 13 29-32

[38] Ginibre J 1970 General formulation of Griffiths' inequalities Commun. Math. Phys. 16 310-28

[39] Álvarez-Nodarse R, Yáñez R J and Dehesa J S 1998 Modified Clebsch-Gordan-type expansions for products of discrete hypergeometric polynomials J. Comput. Appl. Math. 89 171-97

[40] Álvarez-Nodarse R, Arvesú J and Yáñez R J 1998 On the connection and linearization problem for discrete hypergeometric $q$-polynomials Preprint (submitted to Meth. Appl. Anal.)

[41] Connett W C, Markett C and Schwartz A L 1993 Product formulae and convolutions for angular and radial spheroidal wavefunctions Trans. Am. Math. Soc. 338 695-710

Gorlich E, Markett C and Stüpp O 1994 Integral formulae associated with products of Bessel functions: a new partial differential equation approach J. Comput. Appl. Math. 51 135-57

Markett C 1989 Product formulae and convolution structure for Fourier-Bessel series Constr. Approx. 5383-404 
[42] Bang J M and Vaagen J S 1980 The Sturmian expansion: a well-depth-method for orbitals in a deformed potential Z. Phys. A $297223-36$

Budzinski J 1985 Application of modified Newman expansion for analytical evaluation of two-centre, twoelectron integrals over Slater-type orbitals Int. J. Quantum Chem. 28 853-60

Kalnins E G and Miller W Jr 1991 Hypergeometric expansion of Heun polynomials SIAM J. Math. Anal. 22 $1450-6$

Ohja P C 1987 The Jacobi-matrix method in parabolic coordinates: expansion of Coulomb functions in parabolic Sturmians J. Math. Phys. 28 392-6

Prabhakar T R and Jain S 1978 On a general class of polynomials relevant to quantum mechanics Proc. Indian Natl Sci. Acad. A 44 103-16

[43] Capelas de Oliveira E 1992 On generating functions Nuovo Cimento B 107 59-64

Mendas I 1993 Generating functions for the product of the associated Laguerre and Hermite polynomials $J$. Phys. A Math. Gen. 26 L93-5

[44] Harriman J E 1992 Electron densities, momentum densities and density matrices Z. Naturforsch. A 47 203-10

[45] Koepf W and Schmersau D 1998 Representations of orthogonal polynomials J. Comput. Appl. Math. 90 57-94 